\title{
In reply to "Letter to the Editor: Fetal ventriculomegaly: Diagnosis, treatment, and future directions."
}

\author{
Jared M. Pisapia ${ }^{1,2}$ • Gregory G. Heuer ${ }^{1,2}$
}

Received: 22 July 2017 / Accepted: 27 July 2017 /Published online: 8 August 2017

(C) Springer-Verlag GmbH Germany 2017

We thank Dr. Guibaud and colleagues for their insightful comments and additional references, which make our manuscript more comprehensive.

We strongly agree that accurate measurements are paramount in the diagnosis and subsequent counseling in fetal ventriculomegaly. The standardized method for ultrasound measurements based on five anatomic or technical requirements put forth by Guibaud et al. is meant to reduce the variation in cerebral ventricular measurements between centers and to decrease the number of false-positive results. We add that fetal magnetic resonance imaging (MRI) provides improved clarity of ventricle borders and the axial plane, which are sources of potential error in ultrasound-based measurements. Also, several anatomic landmarks that do not change position with the degree of ventricular enlargement, such as the parieto-occipital sulcus, are readily identified on MRI. In addition, segmentation techniques applied to fetal MRI yield volume mea-

Jared M. Pisapia

Jared.Pisapia@uphs.upenn.edu

1 Department of Neurosurgery, University of Pennsylvania, 3400 Spruce Street, 3rd Floor Silverstein Pavilion, Philadelphia, PA 19104, USA

2 Division of Neurosurgery, The Children's Hospital of Philadelphia, Philadelphia, PA, USA surements of all cerebrospinal fluid spaces, which may provide useful prognostic information when combined with currently used linear measurements.

As stated by Drs. Guibaud and Lacalm, the diagnostic algorithm they put forth to determine the etiology of ventriculomegaly based on ultrasound imaging patterns may be similarly applied to MRI. We agree that gathering information related to the underlying pathology of ventriculomegaly is useful for counseling. Furthermore, we concur that the term "severe," in regard to ventricular dilation, is a historical term used for classification and should not be used for prognostic purposes, especially when counseling families. We add that the outcome depends both on the severity of ventriculomegaly as well as associated anomalies.

The commentary and additional references included by Drs. Guibaud, Lacalm, and Rault highlight the complexity and nuances involved in the prenatal evaluation of fetal ventriculomegaly, an understanding of which will enable practitioners to provide the highest level of counseling and care to patients.

\section{Compliance with ethical standards}

Funding source Pediatric Hydrocephalus Foundation Research Grant.

Financial disclosure The authors have no financial relationships relevant to this article to disclose.

Conflict of interest The authors have no conflicts of interest to disclose. 